\title{
The Research of Optimal Length of Left Turn with U-turn at Intersection Based on Statistical and Simulation Analysis
}

\author{
Gan Lü ${ }^{\text {a }}$, Yuanxun Zheng ${ }^{\mathrm{b} * \#}$ and Lihua Xue ${ }^{\mathrm{c}}$ \\ School of Water Conservancy and Environment, Zhengzhou University, Zhengzhou 450002, China \\ a531885766@qq.com, byxzheng@zzu.edu.cn, c1059546488@qq.com \\ *The corresponding author
}

Keywords: Traffic engineering; Intersection; Far lead turn; Simulation analysis; Statistical analysis; Cptimal length; Travel time

\begin{abstract}
Distant lead is one of the ways to eliminate the left turn of the intersection, and the determination of the length of the U-turn is the key to determining the success of the method. For the determination of the optimal length of the far-lead turn-around, the main traffic parameters are determined on the basis of the existing statistic data, and then the VISSIM is used to analyze the intersection in peak period and no-peak period. Finally, the simulation results are statistically analyzed by MATLAB, and the shortest distance of the simulation travel time is taken as the optimal lead length. The results show that the optimal length of the far-lead is obtained in the peak period and the peak-peak period. Under the hypothesis parameter, the optimal length of the U-turn is $300 \mathrm{~m}$ in the peak period and $250 \mathrm{~m}$ in the non-peak period; through theoretical analysis, the peak length of the peak is $297.61 \mathrm{~m}$, and the peak length of $254.21 \mathrm{~m}$.
\end{abstract}

\section{Introduction}

With the rapid development of urban transport, car ownership increased year by year, large and medium cities in the traffic pressure increasing [1]. In the city, the intersection is often the bottleneck of urban transport, but also prone to traffic congestion section. In order to solve the problem of congestion at urban intersections, it is widely used to prohibit the left turn of traffic at intersections. Prohibiting the left turn of the traffic organization program refers to the use of traffic channelization of the idea, from the management means to the intersection of the left side of the intersection of traffic to eliminate and reduce the intersection of the conflict point[2]. The proportion of left-turn traffic at the intersection of the intersection has an important effect on traffic capacity of the intersection, and the proportion of the left-turn traffic at the intersection is reduced to increase the capacity of the intersection $[3,4]$. In order to eliminate the left turn traffic and can turn left the vehicle can quickly reach the destination, the scholars put forward a lot of ideas, including three-dimensional, alfalfa leaf level and far from the first turn. Far to turn the idea is to turn left turn traffic into a straight turn U-turn mode, the left turn traffic to the road section to achieve indirect turn left [5]. At home and abroad, there are many studies on the far-off, including the study of the U-turn and so on. However, the research methods are deduced by the formula or only by simple simulation [6].After simulation and statistical analysis, the length of the research needs to be carried out.

As the signal intersection in time to avoid a part of the left turn traffic generated by the conflict point, it also has a greater impact on vehicle travel time at the signal intersection phase and timing [7]. Therefore, the research on the U-turn pitch of the far-off head is mainly based on the MATLAB mathematical statistical analysis software, which makes reasonable assumptions on the parameters and then uses the PTV-VISSIM micro simulation software to simulate the different U-turn length of the no-signal crossroads Time, and then through the MATLAB software for data analysis, fitting the data curve to obtain the optimal U-turn. 


\section{The Initial Design of the U-turn}

In the urban intersection, the proportion of left turn traffic is an important factor influencing the traffic capacity of the intersection [8]. Turn left traffic is the main factor in the conflict. In the four-way intersection without signal control, it can be seen from the four-way intersection traffic streamline (Figure 1) that there are 16 intersecting points at the intersection of the left-turn traffic, and 16 are divided points. The intersection point generated by the left turn is 12 , occupy $3 / 4$. Through preliminary analysis, the original intersection of traffic conditions will be greatly improved by removing the intersection of left turn traffic flow, which effectively alleviates traffic congestion at intersections.

The Motor Vehicle Turn Left. There is a large number of studies on the elimination of left turn, they are generally divided into a comprehensive ban on the left or part of the way to prohibit left turn on the left traffic flow drainage. Which U-turn is also a way to prohibit the left line, It can be divided into part of the prohibition of the left line and a total ban on the left line, that is the four-way intersection only prohibits a pair of opposite lanes to the left Turn left the imported vehicle. Its basic design idea is to prohibit the vehicle turn left from the management means, that is, to guide the left turn traffic through the intersection of straight line to a certain length when the U-turn and then turn right to achieve the purpose of turning the vehicle (Fig. 2).

Non-motor Vehicle Turn Left. In the city road intersection in the passage of the main consideration is the motor vehicle right, non-motor vehicle turn left mainly through crossing the zebra twice. In the city road intersection--n in the passage of the main consideration is the motor vehicle right, non-motor vehicle turn left mainly through the zebra crossing twice[9,10]. The schematic diagram is shown in Fig. 3.

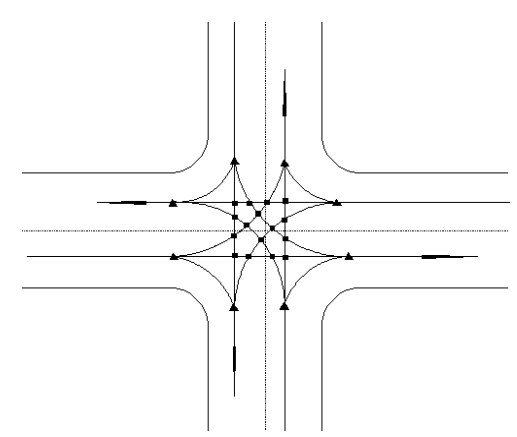

Figure 1. Traffic flow diagram

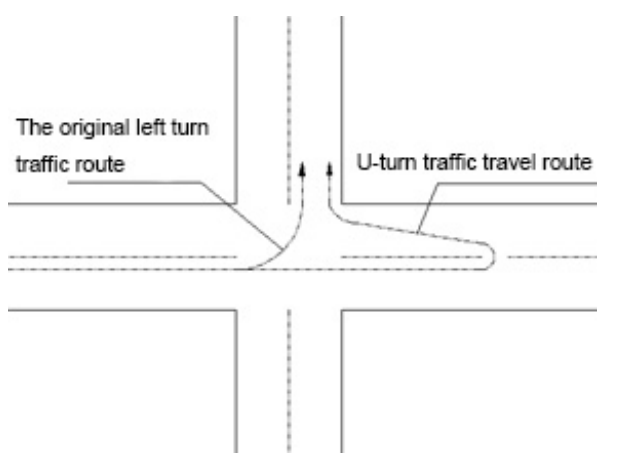

Figure 2. Vehicle line comparison 


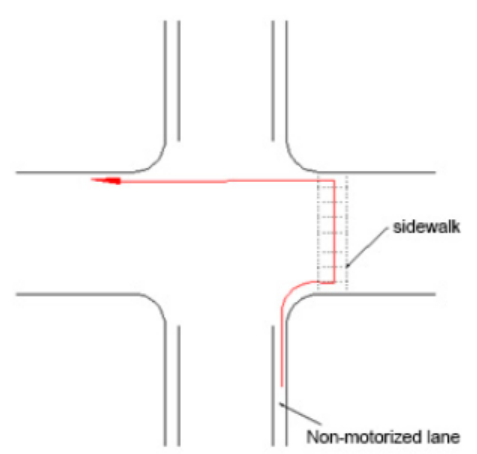

Figure 3. No-motor vehicle

\section{Analysis of U-turn Design and Parameter Assumptions}

The Essential of U-turn Design. In the U-turn design of the main points include two parts:1) the determination of the length of the U-turn; 2) the determination of the opening form. In this paper, we mainly discuss the influence of the length of the nozzle on the intersection traffic. If the distance from the main intersection is too long, the length of the main intersection will affect the traffic at the main intersection. As the rules of the country's turn left, the rules will turn left at the main intersection. , If the opening position is too far from the main intersection position, the left turn time will increase due to the increase in driving distance, the driver may violate the provisions at this time to turn left directly instead of turning of through the U-turn.

U-turn Design Parameters and Parameter Assumptions. There are many factors that affect the length of the far-off, which mainly includes the traffic volume and traffic composition, the intersection of the number of lanes, turn left the number of vehicles and the intersection speed ${ }^{[11]}$.

Assumptions of Traffic Volume and Traffic Composition. The intersection of urban traffic is often difficult to determine due to uncertain factors, and its value is closely related to time. It can be assumed that the hourly design traffic volume is chosen as the basis for calculating the length of the nozzle. But the composition of the vehicle at the intersection has general rules to follow, the vehicle composition to maintain a fixed range of small fluctuations within the fixed value. As the traffic in the VISSIM software traffic vehicles will be divided into cars, trucks, large passenger cars, so according to the China Statistical Yearbook[12] can be summarized in the annual registration of road vehicles in the number of passenger and cargo and the use of MATLAB fitting graphics (Fig. 4) close to the $\mathrm{S}$-curve, that is, passenger and cargo ratio closer to a fixed value.

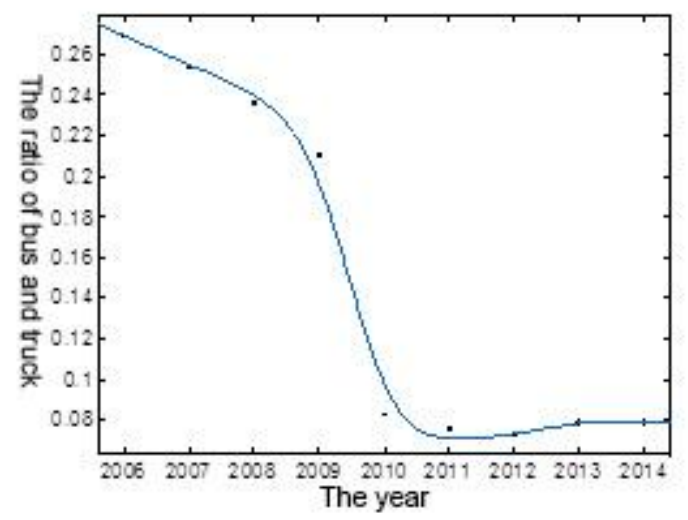

Figure 4. The ratio of cars and trucks changes over time

Statistical analysis of the proportion of various vehicles in civil vehicles expect operating 
vehicles, the initial analysis of the proportion of large passenger cars and large trucks in line with the S-type distribution, the fitting effect shown in Figure 5. Through the fitting can reflect that the civilian vehicle bus and the ratio of large trucks is also similar to the trend of operating vehicle passenger and cargo ratio in recent years, the ratio approaches a constant, it can be used to operate vehicles and civilian vehicle data obtained by the combination of Bus and truck ratio as the design of the selected bus and truck ratio, to determine the ratio of 0.14 。

From the analysis of the data reflects the bus and the proportion of large trucks gradually reduced, the proportion of cars increased year by year, due to the proportion of passenger cars and trucks have been determined, we only need to fit the proportion of the car and the fitting results are shown in Figure 6, combined with the reliability of $92.27 \%$. According to the results of the fitting can be determined in recent years, the proportion of cars is about $95 \%$.

From the above analysis, it can be assumed that in the U-turn design the composition of the traffic is: Car: Bus: Truck $=155: 1: 7$.

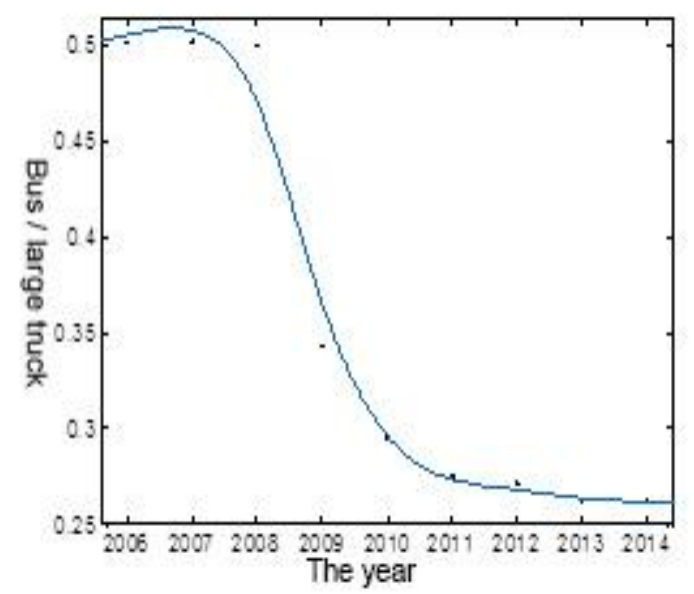

Figure 5. The ratio of buses and trucks changes over time Figure

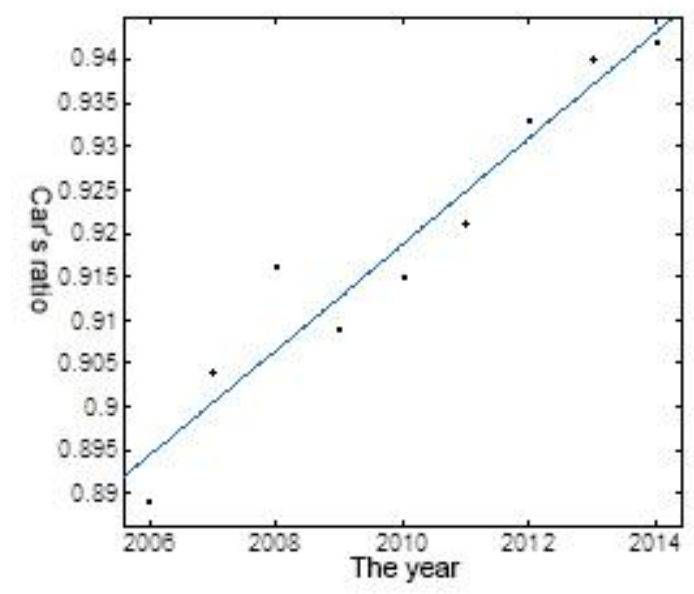

Figure 6. The ratio of cars changes over time

Assumptions about the Number of Intersections Lanes. In the non-signal-controlled U-turn intersection design in order to ensure that the interference between the vehicles as small as possible, that is, the vehicle turn left, straight and turn right without interfere with each other and meet the minimum land condition, the number of lanes at the intersection is generally meet the smallest demand that vehicles in each direction occupy a lane, the number of lanes is two-way six-lane.

The number of lanes at the city intersections should also match the number of lanes of the import and exit sections to ensure that the city intersections do not become a traffic bottleneck. According 
to the "Urban Road Traffic Planning and Design Code"(GB50220-95)[13], the road width of the urban road network can be obtained in Table 1.

Table 1 Index of the roads width

\begin{tabular}{|c|c|c|c|c|c|c|}
\hline \multirow{3}{*}{$\begin{array}{l}\text { project } \\
\text { Road width (m) }\end{array}$} & \multicolumn{6}{|c|}{ City size and population Freeway Trunk road Secondary road Slip road } \\
\hline & \multirow{2}{*}{ Big } & & $40-45$ & $45-55$ & $40-50$ & $15-30$ \\
\hline & & $<2 \mathrm{n}$ & $35-40$ & $40-50$ & $30-45$ & $15-20$ \\
\hline & Medium & city & - & $35-45$ & $30-40$ & $15-20$ \\
\hline
\end{tabular}

According to the population of more than 200 million population of the main road of its road width of $45-55 \mathrm{~m}$, secondary road to $3.75 \mathrm{~m}$, and three road lane width of $2.5 \mathrm{~m}$, non-motorized road for the one-way $4.5 \mathrm{~m}$, sidewalk for the one-way $0.75 \mathrm{~m}$, the middle belt with a minimum width of $2.5 \mathrm{~m}$, both sides of the minimum width of $2.25 \mathrm{~m}$ [14]. Two-way six-lane road width is: $\mathrm{W}=3.75 \times 6+4.5 \times 2+0.75 \times 2+2.5+2.25 \times 2=40 \mathrm{~m}$ it meets the lowest limitation of the urban secondary road. Therefore, the two-way six-lane road is also the minimum number of lanes to meet the required road width requirements.

The Number of Turn Left Vehicles and the Intersection Speed. The number of revolving vehicles is usually random at the intersection, and its numerical size is often closely related to the geographical location of the intersection. It should be designed according to the specific location. In this paper, it is assumed that the intersection is in four directions The same volume can be selected, and several sets of left turn traffic volume can be selected for simulation analysis, so as to analyze the influence of the left turn traffic on the optimal length of the far nozzle.

The general traffic speed of the vehicle at the intersection is often slowed down to the intersection, and the actual driving speed of the vehicle at the intersection is often smaller than that of the road. In the urban road, the design speed of the internal road in the big city is generally $40-60 \mathrm{~km} / \mathrm{h}$ Medium city internal road design speed is generally $30-40 \mathrm{~km} / \mathrm{h}$, Small city internal road design speed is generally $20-40 \mathrm{~km} / \mathrm{h}[14]$. The problem of congestion at urban intersections is usually found in large and medium-sized cities. In this paper, the design speed of urban roads is chosen as the basis of design. Assuming that the driving speed of the car at the intersection is $30 \mathrm{~km} / \mathrm{h}$, The truck speed will be reduced accordingly, the use of truck speed $25 \mathrm{~km} / \mathrm{h}$, Bus speed $20 \mathrm{~km} / \mathrm{h}$.

\section{VISSIM Simulation}

In this study, the optimal length in the study is taken to simulate the peak time and flat peak periods of the road network. In the simulation, it is assumed that the influence of the non-motor vehicle is taken into account. The traffic volume of the simulation is based on the load of the road network assuming the use of two kinds of road network traffic as shown in the following Table 2.

Table 2 Traffic flow of two circumstances

\begin{tabular}{lllll}
\hline Project & East import & West import & South import & North import \\
\hline Peak Period $(\mathrm{veh} / \mathrm{h})$ & 1200 & 1200 & 1000 & 1000 \\
Non-peak period $(\mathrm{veh} / \mathrm{h})$ & 1000 & 1000 & 700 & 1000 \\
\hline
\end{tabular}

In this simulation, starting from the intersection $100 \mathrm{~m}$, every $50 \mathrm{~m}$ set far away from the head, until $400 \mathrm{~m}$, the seven sets of simulation data, according to the statistics to get the best U-turn length.

In this paper, we mainly test the left and right travel time of two imported roads and do statistical analysis according to the average number of test data and then determine the optimal length in order to provide reliable research methods for the city road intersection design.

Simulation Analysis of Peak Period. In the case of traffic congestion simulation analysis, when the U-turn exit from the near intersection will appear in the far away from the head to wait for the 
length of the vehicle turned to the original intersection of the normal flow of the vehicle, resulting in the intersection of traffic conditions vicious cycle, In this simulation, we set U-turn exit at the $100 \mathrm{~m}$ and the condition come out that the vehicle can not complete the whole process of simulation (Fig。 7). The simulation data of the 7 group are shown in Table 3.

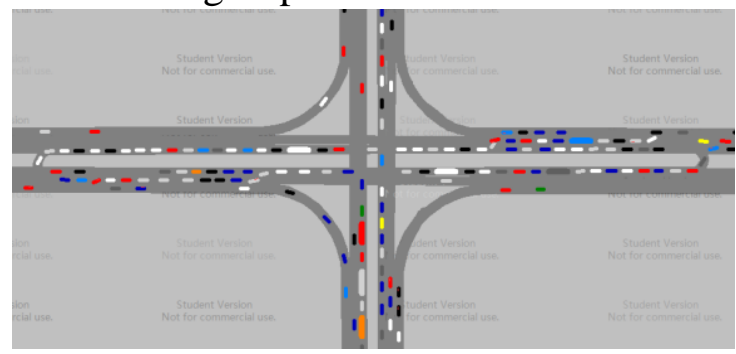

Figure 7. Simulation of 100 meter

Table 3 Travel time of 100-400 meters simulation

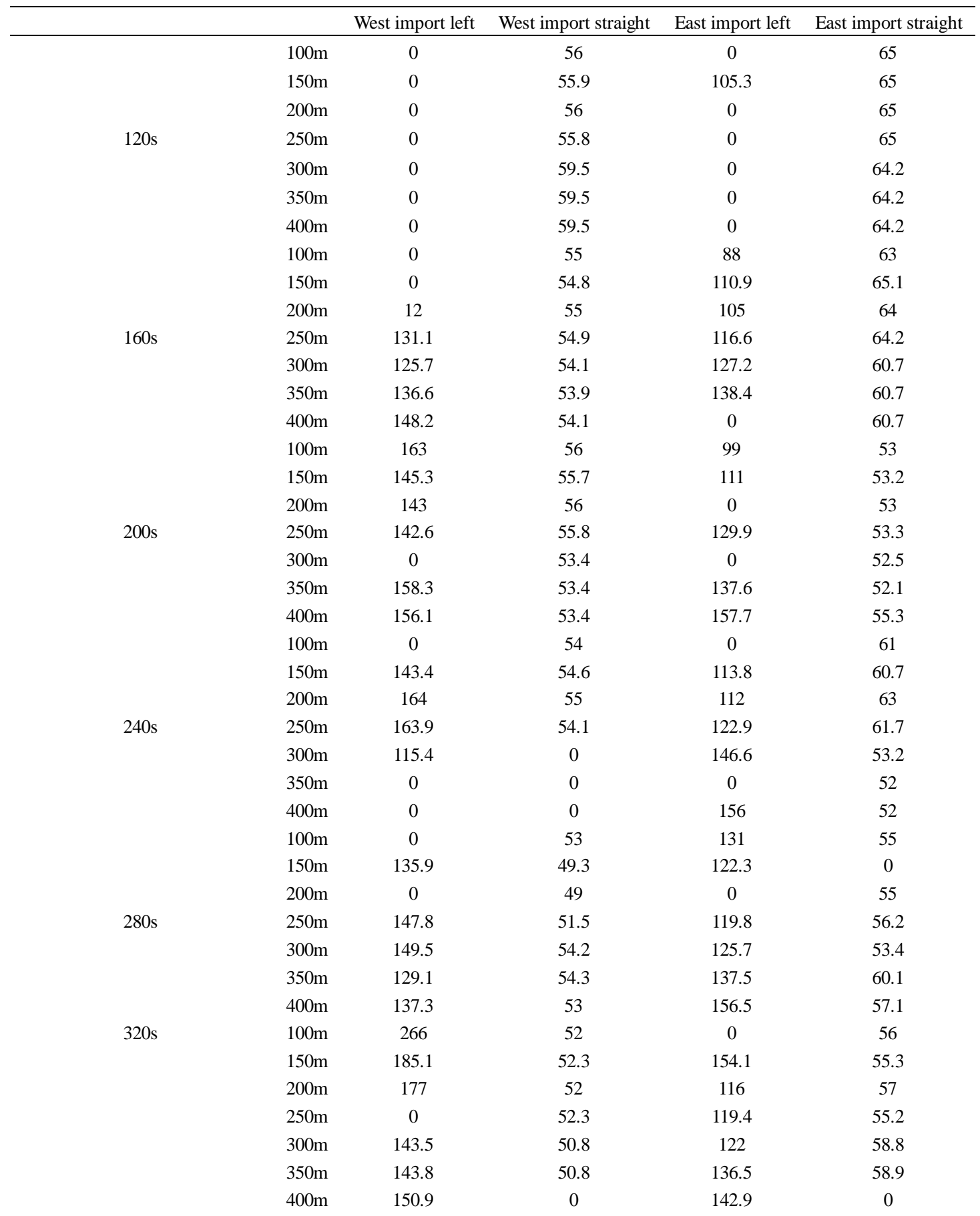




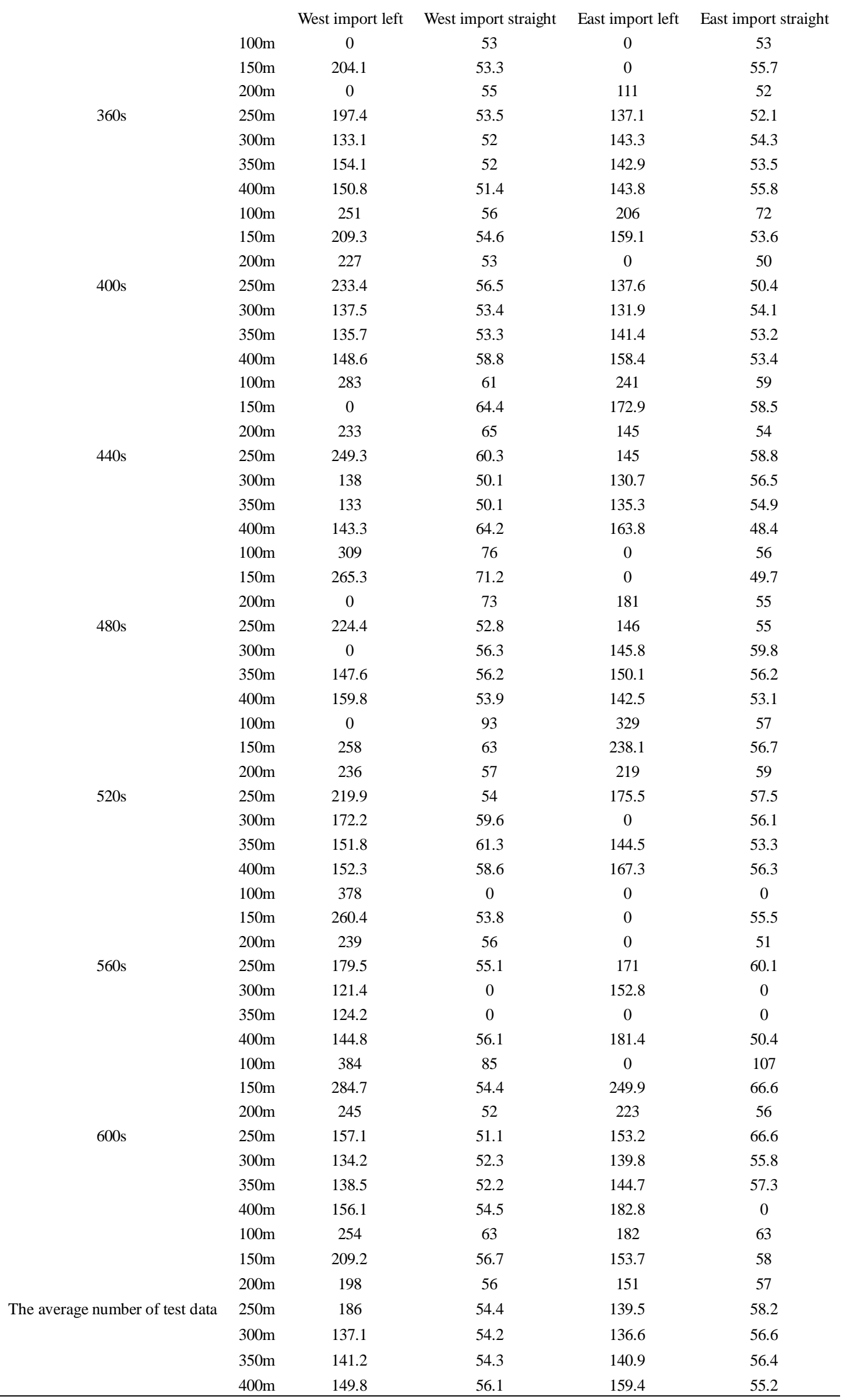

Note: (1)The time point in which 0 appears in the simulation data indicates that the vehicle has 
not been detected and the average is not counted ;

(2)In the simulation of the first $100 \mathrm{~s}$, the vehicle did not enter the intersection, the data detection was incomplete and the data was recorded from $120 \mathrm{~s}$;

(3)The travel time in the table is in seconds (s).

A preliminary analysis of the left-turn simulation data shows that the travel time begins to decrease with the increase of the long-lead length, the travel time increases with the increase of the length of the long lead when the length is further increased, it can be seen that there is a minimum point in the travel time, the far-off length corresponding to the minimum point is the optimal length. A preliminary analysis of the simulation data can be seen, in the traffic congestion, the influence of the length of the left hand on the straight vehicle is not obvious, the travel time varies with the length of the distance. Therefore, we need to count and analyze the travel time of the vehicles turning left at the two entrances, the results are shown in Fig. 8 and Fig. 9.

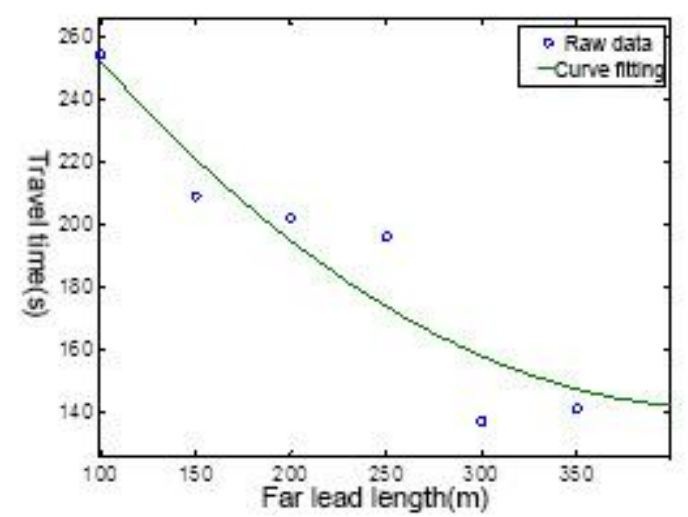

Figure 8. Curve fitting of west entrance in peak period

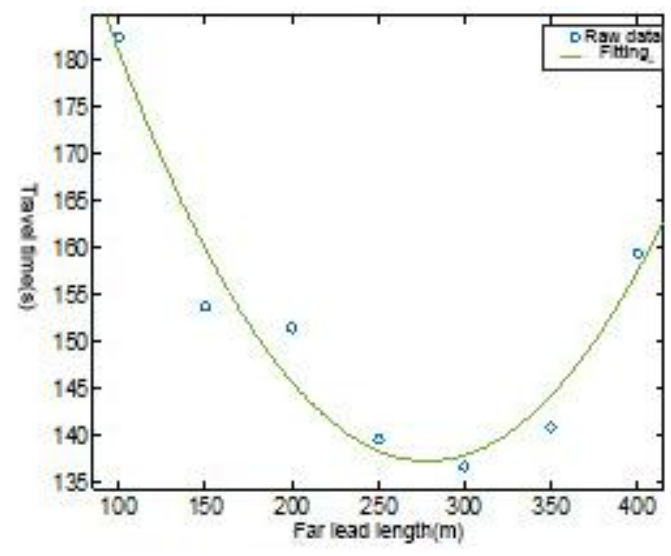

Figure 9. Curve fitting of east entrance in peak period

From fig. 8, we still can not clearly see the best long-lead length of turning left in west, but we can clearly find the optimal length of the east entrance to the left from fig. 9. However, the total optimal length should not be determined by fitting the two entrances separately but be determined using the sum of the two averages, as shown in the following table 4.

Table 4 Total average travel time in peak period

\begin{tabular}{lccccccc}
\hline $\begin{array}{l}\text { Far lead length } \\
(\mathrm{m})\end{array}$ & 100 & 150 & 200 & 250 & 300 & 350 & 400 \\
\hline Travel time(s) & 437 & 363 & 350 & 326 & 274 & 282 & 309
\end{tabular}




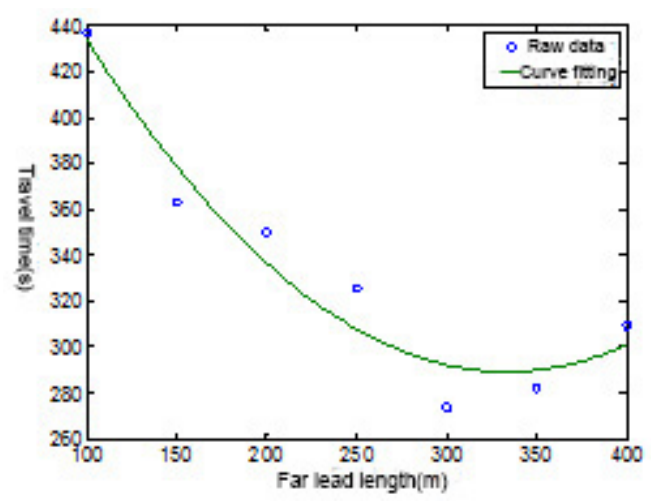

Figure 10. Curve fitting travel time between far lead length with total average travel time

From the general average travel time fitting curve, we can clearly see that the best far-off head length is around $300 \mathrm{~m}$ in the case of traffic.

Simulation Analysis of Non-peak Period. Simulation analysis in the case of non-peak time(figure11), The distance between the U-turn's exit and the intersection is not near when the length of the vehicle waiting for the U-turn is turned off, affecting the normal driving of the original intersection, and the same is true for the seven hours of simulation, The fitting curves are shown in Fig. 12 and Fig. 13.

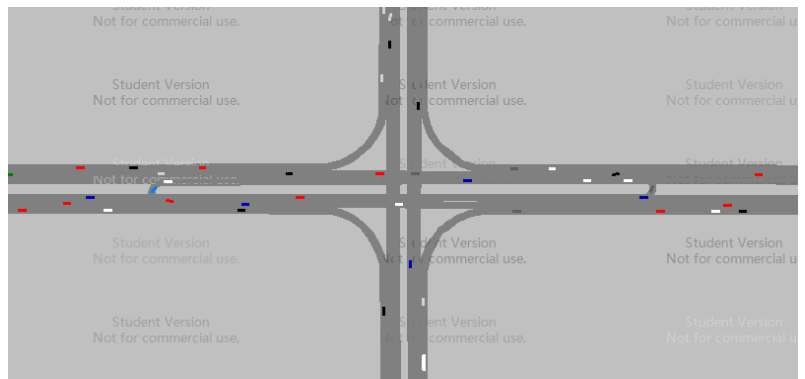

Figure 11. Simulation of non-peak period

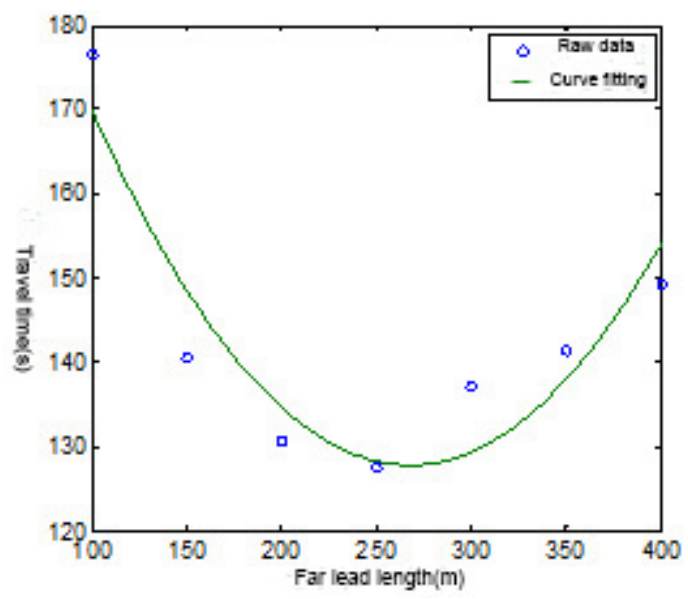

Figure 12. Curve fitting of west entrance in non-peak period 


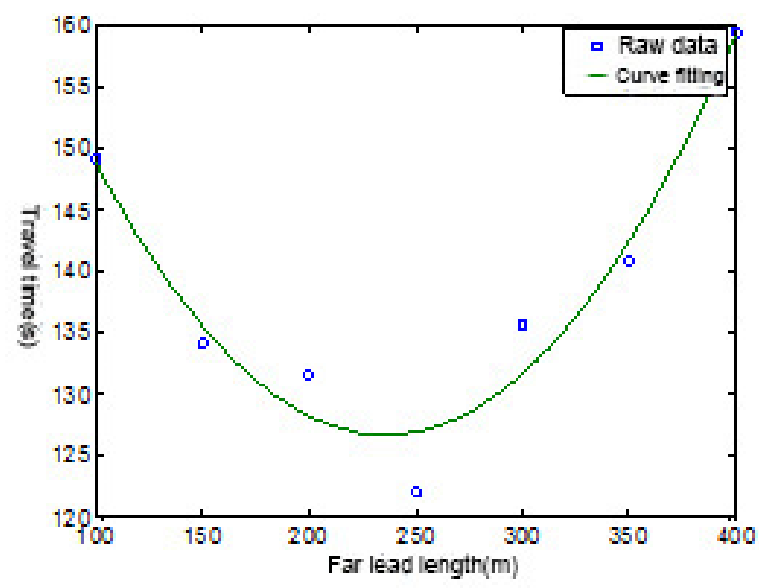

Figure 13. Curve fitting of east entrance in non-peak period

From the above two entrances for the individual fitting can be seen in the case of smooth traffic in the case of two into the mouth of the best length of the left are more obvious, the optimum length is about $250 \mathrm{~m}$, but the actual situation needs to be determined based on the total travel time of the two entrances, the total travel time is shown in Table 5, the total travel time fitting curve is shown in Fig. 14.

Table 5 Total average travel time in non-peak period

\begin{tabular}{lccccccc}
\hline Far lead length $(\mathrm{m})$ & 100 & 150 & 200 & 250 & 300 & 350 & 400 \\
& & & & & & & \\
\hline Travel time(s) & 326 & 275 & 262 & 250 & 273 & 282 & 309 \\
\hline
\end{tabular}

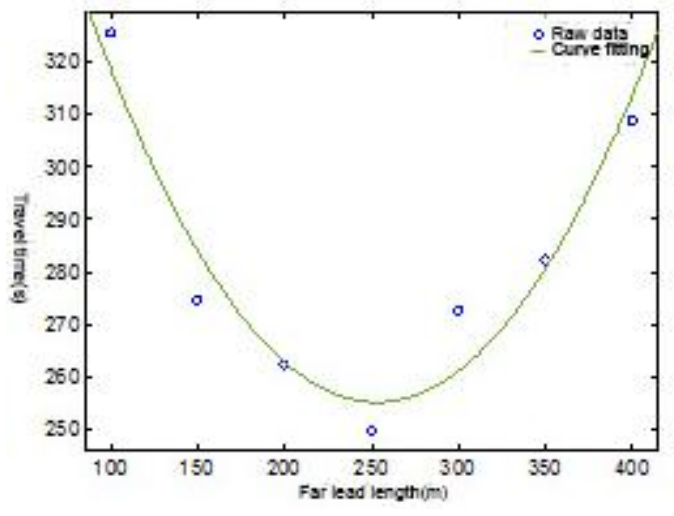

Figure 14. Curve fitting of travel time

As can be seen from Fig. 14: In the case of non-peak hours, the total travel time and the fitting curve have the same frequency as the two fitting curves, the optimum length is about $250 \mathrm{~m}$.

According to Ronglong Zhao [15] Model based on accident rate studies show that the six-lane U-turn spacing should be not less than $137 \mathrm{~m}$, using the formula in the study of Zhirui Yan [16]. Using the parameters used in this paper, the long-term length of the peak period should be slightly larger than $297.61 \mathrm{~m}$, and the length of the peak period should be slightly larger than $254.21 \mathrm{~m}$, this is a strong consistency with the results of this study. 


\section{Conclusion}

Based on the statistical analysis of the existing statistical data, this paper determines the traffic composition of the intersection. Then, analyzing the traffic conditions based on the identified traffic conditions and dividing the traffic situation into two cases: traffic congestion and traffic unobstructed. The data is processed statistically by the MATLAB software to obtain the best far-off head length in each case. The main findings are as follow:

(1)Based on the relevant assumptions and input parameters, the optimal long-lead length at the intersection of peak time is about $300 \mathrm{~m}$, and the best long lead length in the peak period is $250 \mathrm{~m}$ according to the simulation and statistical analysis. Theoretical research is consistent. In the actual intersection, the distance between the actual intersection can be obtained according to the number of lanes, traffic composition and traffic volume of the actual intersection. The same method can be used to get the optimal length of the corresponding intersection. Fence length fixed at peak hours to increase the fence and other measures to achieve long lead length changes;

(2)In this paper, the method of determining the optimal length of the far-off is combined with the simulation analysis and statistical analysis. The optimal long-lead length is of some theoretical value and engineering significance. In the actual project, its traffic situation and road conditions through the simulation analysis and statistical analysis of the corresponding intersection of the optimal program for the urban road intersection design to provide technical support.

(3)In this study, we consider the factors such as road width and traffic flow but do not take into account the driver's behavior factors and the adaptability of software to our traffic situation. In the next study, we will take full account of the impact of these factors on the length of the far-off.

\section{Acknowledgments}

This work was supported by Natural Science Foundation of Henan (Grant Nos. 51508114) and Science and Technology Program of Communications Department of Henan Province (No. 2014K37-2), the authors are very grateful to the test field support of Communications Department of Henan Province. The authors thank the advice and support of Dr. Pan Ernian at The University of Akron. Special thanks are extended anonymous reviewers for their value comments.

\section{References}

[1] L Gan, P Zhang and D.H Duanmu, Building Material and Civil Engineering, (Guilin,China, August 15-16, 2015). Vol.1, p.746-750.

[2] K.J Long, Q Chen, Journal of Beijing University of Technology, vol.37 (2011) No.6, p.876-881.(in Chinese).

[3] X.H Chen, P.Y Ye, Journal of Tongji University(Natural Science),vol.36 (2008) No.8, p.1067-1072.(in Chinese).

[4] Y.X Zheng, T Li, Journal of Zhengzhou University(Engineering Science), vol.37 (2016) No.2,p.87-91.(in Chinese).

[5] Z Guo, J.Y Chen and W.J Wang; Technology \& Economy in Areas of Communications, vol.10 (2008) No.6,p.60-62.(in Chinese).

[6] N Zhang, K Chen and Z.H Gao, et al: Journal of Traffic and Transportation Engineering, vol.8(2008)No.1,p.78-82.(in Chinese).

[7] J.Q Leng, Y.Q Leng, Y.P Zhang, Journal of Kunming University of Science and Technology(Science and Technology), vol.34(2009)No.3,p.56-59.(in Chinese).

[8] H Wang, W Wang and $\mathrm{J}$ Chen, Journal of Huazhong University of Science and Technology(Nature Science Edition), vol.35(2007)No.1,p.114-117.(in Chinese). 
[9] X.G Yang, J Yang and Y.Q Shi, Urban Transport of China,vol.10(2012)No.4, p.65-71.(in Chinese).

[10]J Shan, W Luo, Road Traffic \& Safety, vol.7(2007)No.3, p.45-51. (in Chinese).

[11]C.Q Shen, Research on Applicability of Right Turn Followed by U-turn of Unsignalized Intersections on First-grade-highway (MS. Shanghai Jiaotong University, China 2013), p. 26-28(in Chinese).

[12] Statistics Bureau of the People 's Republic of China: China Statistical Yearbook 2015, 2015.(in Chinese).

[13] Ministry of Construction of the People's Republic of China: Code for design and design of road traffic in the city of the People 's Republic of China , Beijing: China Building Industry Press, 1995 .(in Chinese).

[14]Ministry of Construction of the People 's Republic of China: Code for design of urban roads (CJJ37-90), Beijing: China Building Industry Press, 1995.(in Chinese)

[15]R.L Zhao, J.J Fan and P Liu, Journal of Southeast University (English Edition), Vol.26(2010)No.4, p.628 -632.

[16]Z.R Yan, Research Cited Left U-turn Median Strip away the Opening Pitch(MS. School of Highway Chang'an University, China 2014),p.49-52(in Chinese). 